\title{
Foundations of the Severe Acute Respiratory Syndrome Preparedness and Response Plan for HealthCare Facilities
}

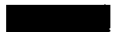 \\ Arjun Srinivasan, MD; Lawrence C. McDonald, MD, MPH; Daniel Jernigan, MD, MPH; Rita Helfand, MD, MPH; \\ Kathleen Ginsheimer, MD, MPH; John Jernigan, MD, MPH; Linda Chiarello, RN, CIC; Raymond Chinn, MD; Umesh Parashar, MD; \\ Larry Anderson, MD; Denise Cardo, MD; SARS Healthcare Preparedness and Response Plan Team*
}

\begin{abstract}
OBJECTIVE: To help facilities prepare for potential future cases of severe acute respiratory syndrome (SARS).

DESIGN AND PARTICIPANTS: The Centers for Disease Control and Prevention (CDC), assisted by members of professional societies representing public health, healthcare workers, and healthcare administrators, developed guidance to help facilities both prepare for and respond to cases of SARS.

INTERVENTIONS: The recommendations in the CDC document were based on some of the important lessons learned in healthcare settings around the world during the SARS outbreak of 2003, including that (1) a SARS outbreak requires a coordinated and dynamic response by multiple groups; (2) unrec-

ognized cases of SARS-associated coronavirus are a significant source of transmission; (3) restricting access to the healthcare facility can minimize transmission; (4) airborne infection isolation is recommended, but facilities and equipment may not be available; and (5) staffing needs and support will pose a significant challenge.

CONCLUSIONS: Healthcare facilities were at the center of the SARS outbreak of 2003 and played a key role in controlling the epidemic. Recommendations in the CDC's SARS preparedness and response guidance for healthcare facilities will help facilities prepare for possible future outbreaks of SARS (Infect Control Hosp Epidemiol 2004;25:1020-1025).
\end{abstract}

Healthcare facilities and healthcare workers (HCWs) were at the center of the 2003 outbreak of severe acute respiratory syndrome (SARS). Reports of unexplained pneumonia in HCWs prompted the initial investigation into the outbreak, ${ }^{1}$ and specimens obtained from a HCW first yielded the causative agent now known as SARS-associated coronavirus (SARS-CoV). ${ }^{2}$ SARS posed a difficult challenge to healthcare facilities from both the impact of healthcare-associated transmission and the resource burden of controlling and preventing further spread. HCWs accounted for 25\% of cases in Hong Kong and $65 \%$ in Canada, ${ }^{3}$ and hospital transmission accounted for $72 \%$ of all cases in Toronto ${ }^{4}$ and $55 \%$ in Taiwan. ${ }^{5}$

As the 2003 outbreak waned, it became increasingly clear that healthcare facilities in the United States needed to take advantage of the important lessons learned in other areas to help prepare for potential SARS outbreaks. Thus, beginning in June 2003, the Centers for Disease Control and Prevention (CDC) began developing guid- ance for healthcare facilities. This guidance was developed with key input from more than 20 partner organizations representing public health and the spectrum of healthcare delivery in the United States, including prehospital care, nursing, physician, laboratory, and healthcare support staff as well as administrators. The resulting SARS preparedness and response plan for healthcare facilities is one component of a broader initiative that provides guidance for community-level SARS preparedness and response. ${ }^{6}$ Throughout development of this plan, it was emphasized that SARS preparedness and response planning should not occur in a vacuum but should both build on and be used as a framework for planning for other potential infectious disease emergencies such as pandemic influenza.

This article summarizes some of the lessons learned during the SARS outbreak of 2003 that served as the foundations for the preparedness and response plan for healthcare facilities and then describes the measures

Drs. Srinivasan, McDonald, D. Jernigan, J. Jernigan, and Cardo and Ms. Chiarello are from the Division of Healthcare Quality Promotion, and Drs. Helfand, Parashar, and Anderson are from Viral and Rickettsial Diseases, National Center for Infectious Diseases. Centers for Disease Control and Prevention, Atlanta, Georgia. Dr. Ginsheimer is from the Maine Bureau of Health, Augusta, Maine. Dr. Chinn is from Sharp Memorial Hospital, San Diego, California.

Address reprint requests to Arjun Srinivasan, MD, Division of Healthcare Quality Promotion, Centers for Disease Control and Prevention, 1600 Clifton Road, MS-A35, Atlanta, GA 30333.

${ }^{*}$ Members of the SARS Healthcare Preparedness and Response Plan Team included S. Solomon, Centers for Disease Control and Prevention, Atlanta, GA: M. Keifer, National Institute for Occupational Safety and Health, Centers for Disease Control and Prevention, Atlanta, GA: $M$. Pearsan, Division of Healthcare Quality Promotion, National Center for Infectious Diseases, Centers for Disease Control and Prevention, Atlanta, GA; J. Bentley, American Hospital Association, Washington, DC; J. Steinberg, Emory University Hospital, Atlanta, GA; K. Woeltje, Medical College of Georgia, Augusta, GA; R. Koppaka, Division of Global Migration and Quarantine, National Center for Infectious Diseases, Centers for Disease Control and Prevention, Atlanta, GA; C. Van Beneden, Division of Bacterial and Mycotic Diseases, National Center for Infectious Diseases, Centers for Disease Control and Prevention, Atlanta, GA; and R. Weinstein, Cook County Hospital, Chicago, IL. 
designed to address these issues. Because of the dynamic and variable nature of a SARS outbreak, response plans must be flexible and should include provisions to escalate measures as an outbreak progresses. In recognition of this, measures in the CDC SARS guidance include a range of proposed responses that depend on the level of SARS activity in both the community and the healthcare facility.

\section{FOUNDATIONS OF THE SARS \\ PREPAREDNESS AND RESPONSE}

GUIDANCE FOR HEALTHCARE FACILITIES

Outbreaks of SARS-CoV in other countries and the limited experience in the United States revealed some important lessons for preparedness and response planning in healthcare facilities. It is these critical "lessons learned" that formed the foundation of the CDC SARS guidance for healthcare facilities.

\section{A SARS Outbreak Requires a Coordinated and Dynamic Response by Multiple Groups}

Triage and management of SARS patients involves many clinical services within the hospital, including emergency departments, outpatient clinics, medical units, intensive care units, radiology, laboratory services, and others. Entry screening and access control efforts will require the assistance of facility security staff. Engineering staff input will be needed to help with air-handling systems. Increased demand for personal protective equipment will require participation of materials management staff. Clear and rapid communication with the public and press will be important and can be facilitated by public relations staff. Training and education on infection control measures will require the involvement of education staff. Finally, healthcare facilities will also need to work closely with local and state health departments so that preparedness and response efforts can be coordinated at the community level. Facilities must be able to escalate response measures quickly, using a graded or stepped approach, as the outbreak becomes more widespread, because recommendations that are appropriate in one setting may not be adequate in another. Although it is not addressed in the current SARS plan, a plan to downgrade response measures quickly and safely is also an important aspect of preparedness that should be considered.

\section{Unrecognized Cases of SARS-CoV Are a Significant Source of Transmission}

The outbreaks of SARS in Toronto and Taiwan were initiated and propagated by exposures to unrecognized cases. In Toronto, 128 cases in the initial outbreak were attributed to exposure to unrecognized SARS-CoV infections in patients, visitors, and HCWs. ${ }^{7}$ The second phase of the Toronto outbreak occurred following exposures to another patient with an unrecognized infection. ${ }^{8}$ In Taiwan, after an initial phase characterized by imported cases and limited secondary transmission, exposure to unrecognized cases led to a rapid escalation of a second phase of the outbreak. ${ }^{5}$ During the 2003 outbreak of
SARS, there were multiple potential sources for unrecognized cases in healthcare facilities, including patients, visitors, and HCWs. Surveillance measures directed at each of these groups, along with contact follow-up, assisted in early case recognition and were critical in terminating the outbreaks of 2003.

\section{Restricting Access to the Healthcare Facility Can Minimize Transmission}

Hospital-acquired infections among patients, HCWs, and visitors accounted for most of the SARS cases in Toronto and Taiwan. ${ }^{4,5}$ In Taiwan, Singapore, and Hong Kong, healthcare-associated transmission preceded and was largely responsible for subsequent increased transmission in the community. This finding suggests that outbreaks may start in hospitals before disseminating to the community. ${ }^{9}$ Therefore, limiting healthcare-associated transmission may be critical in terminating an outbreak at the earliest stages. In the 2003 outbreak, controlling hospital access by limiting the number of entrances facilitated screening of healthcare providers and visitors. ${ }^{10}$ Additionally, limitations on visitors, nonessential staff, and new admissions to facilities in communities where transmission was occurring helped limit the number of individuals who might have been potentially exposed to SARS. ${ }^{10,11}$

\section{Airborne Infection Isolation Is Recommended, But Facilities and Equipment May Not Be Available}

Experience to date indicates that most SARS transmission occurs from direct patient contact or exposure to large respiratory droplets and that droplet precautions can prevent most transmission. ${ }^{12,13}$ However, there are reports in which airborne spread cannot be ruled out, ${ }^{14-16}$ leading to the recommendation that airborne infection isolation be used for patients who are, or are suspected to be, infected with SARS-CoV. Because airborne infection isolation rooms (AIIRs) are normally needed for only a select group of conditions, most healthcare facilities have limited numbers. In 2003, availability of these rooms was quickly exceeded in areas with significant SARS activity. Similar challenges would likely occur in the United States if communities were faced with large numbers of patients requiring airborne infection isolation. A small study of hospitals in the midwestern United States found that few facilities had AIIRs in intensive care units, and none had such rooms in their emergency departments. ${ }^{17}$ In a recent survey of infectious disease specialists who participate in the Emerging Infections Network of the Infectious Diseases Society of America, 29\% of respondents indicated there were no AIIRs in their emergency department, and $17 \%$ indicated there were no AIIRs anywhere in their facility. ${ }^{18}$ This same survey revealed that supplies of appropriate respirators for airborne isolation (N-95 or higher masks) may also pose a challenge, as $25 \%$ of respondents indicated their facility had experienced a shortage of respirators during the outbreak, despite the limited number of cases in the United States. ${ }^{18}$ 


\section{Staffing Needs and Support Will Pose a Significant Challenge}

Strict adherence to infection control precautions is essential in limiting transmission to HCWs. ${ }^{19,20}$ Staff members will need rapid education and training on the use of personal protective equipment and may require additional emotional and logistical support. ${ }^{10,19}$ Likewise, wearing extensive personal protective equipment, especially particulate respirators, for prolonged periods of time and the need for careful attention to how that equipment is donned and removed are exhausting for $\mathrm{HCWs} .{ }^{10} \mathrm{HCW}$ fatigue, combined with increased triage and surveillance measures, may require higher staffing levels. At the same time, the availability of healthcare personnel may actually decrease due to furloughs for illness and exposures, as evidenced by even the limited SARS experience in the United States in $2003 .{ }^{21}$

\section{CDC SARS GUIDANCE FOR HEALTHCARE FACILITIES: PREPAREDNESS PLANNING}

The most effective systems for controlling an outbreak are those that are developed and tested before an outbreak occurs. Given this, and the fact that responding to a SARS outbreak will require flexible plans and coordination of numerous groups, both inside and outside a healthcare facility, the guidance document recommends that facilities develop a formal, written SARS preparedness and response plan. Development of such a plan will help to not only ensure that all issues and needs have been addressed, but also reduce panic and confusion if an outbreak occurs by clearly defining roles and responsibilities.

It is recommended that the facility designate a "SARS coordinator" who has both technical expertise in infection control and outbreak management as well as decision-making authority to lead the planning efforts. As the individual most familiar with the plan's components, this coordinator would also be in an ideal position to direct an outbreak response should it become necessary. SARS planning must be undertaken by a multidisciplinary group that includes representatives from all areas that would potentially be affected by an outbreak, including patient care, administrative, and support staff. The committee may also need adjunct members to help deal with specific issues such as the role of students and trainees during a SARS outbreak and risk management. Ideally, the planning committee will also include a representative from the local or state health department who can help the facility coordinate planning efforts with the broader community. Having a representative from the health department will also help ensure effective communication between the facility and the health department. SARS planning should not occur in a vacuum but rather should build on existing preparedness and response plans for bioterrorism or other infectious disease emergencies and should be addressed by the same groups responsible for developing those plans. The planning committee will need to consider all aspects of a SARS response, including administrative, environmental, and communication measures, and the individual work practices required to detect the introduction of SARS-CoV, prevent its spread, and manage the impact on the facility and the staff.

\section{CDC SARS GUIDANCE FOR HEALTHCARE FACILITIES: RESPONSE ACTIVITIES}

The presence of SARS in the community or facility will prompt the initiation of response activities. Although specific response measures will change as an outbreak progresses, general response activities recommended in the guidance document are driven by the important lessons learned in healthcare facilities during the 2003 SARS outbreak.

\section{A Respiratory Hygiene and Cough Etiquette Policy May Serve as a First Line of Defense}

Once an outbreak of SARS is recognized, it becomes easier to identify patients who have appropriate risk factors and consistent signs and symptoms. However, it is much more difficult to identify cases of SARS prior to the recognition of an outbreak, as epidemiologic risks will be less well defined and symptoms are nonspecific and far more likely to represent other respiratory illnesses.

Given the challenge of recognizing early cases of SARS and considering the potential for spread of other respiratory infections in healthcare settings, contributors to the CDC SARS guidance recommended a broader strategy to prevent healthcare-associated transmission of respiratory illnesses. Based on studies of SARS transmission, it appears that measures designed to control respiratory droplets and secretions along with hand hygiene would offer significant protection to other patients and HCWs who have close contact with source-patients. ${ }^{12,13}$ Beyond SARS, these measures would also help prevent the transmission of many other important pathogens that are spread by the droplet route, such as influenza and Mycoplasma pneumoniae. ${ }^{22}$ Controlling source-patient secretions has also been recommended as a control strategy for tuberculosis, a pathogen that can be spread by the airborne route. ${ }^{23}$

The CDC healthcare facility guidance describes a new approach to managing patients with febrile respiratory illness, which has been termed "respiratory hygiene/cough etiquette." Elements of this strategy have been in routine use at some pediatric centers for controlling annual outbreaks of respiratory syncytial virus and other infections. ${ }^{24}$ The approach includes the following:

1. Patients with febrile respiratory illness should be encouraged to report their symptoms when they arrive for care.

2. Patients with active symptoms should be instructed to practice frequent hand hygiene, especially after touching their face, and to cover their nose and mouth to help control secretions. They could be given a surgical mask or facial tissues to accomplish this. Patients will also need access to waste receptacles and hand hygiene supplies. 
3. These patients should be separated, as possible, from other patients in the waiting area.

4. Signs should be posted in the waiting areas to educate patients about these measures.

5 . While in the healthcare setting, these patients should be cared for using droplet precautions until it is determined that the cause of their symptoms does not require such precautions.

\section{Surveillance and Screening Measures}

Surveillance efforts will need to be coordinated with local and state health departments as outlined in the surveillance section of the guidance document (supplement B). To limit potential exposures to unrecognized cases in the healthcare setting, CDC guidance for healthcare facilities specifically emphasizes that surveillance and screening plans for early case recognition be directed at visitors, patients, and $\mathrm{HCWs}$.

Visitor Screening. Points of entry to a healthcare facility are a logical place to initiate surveillance for SARS cases. When there is SARS activity in the world but none in the local community, entry screening could be passive, such as signs posted at entrances alerting entrants to the epidemiologic risks and symptoms of SARS and requesting that those with both risks and symptoms alert facility staff. If and when SARS activity increases and transmission is occurring in the catchment area around the facility, active entry screening should be considered. Triage stations should be placed at hospital entrances where all entrants, including visitors and employees, can be screened for any potential exposures to SARS patients and evaluated for symptoms. Adjunct measures, such as limiting the number of entrances to the facility and the number of visitors, may help facilitate screening.

HCW Surveillance. HCWs also represent a potential source of unrecognized SARS cases and should be carefully monitored as early detection of SARS-CoV disease in HCWs will help reduce transmission. When the number of SARS cases cared for in a facility can be managed with existing staff and facility resources and there is no evidence of transmission in the facility, surveillance measures could be limited to those $\mathrm{HCW}$ with direct contact with patients infected with SARS-CoV. However, once transmission occurs in the facility or when there are a larger number of SARS patients receiving care, surveillance should be expanded to all HCWs in the facility regardless of whether they have had known contact with SARS patients. Facilities will need to work closely with health departments to coordinate surveillance of HCWs who are furloughed or who present to other facilities for care. Surveillance measures should include active monitoring of HCWs for symptoms with temperature checks and questionnaires regarding respiratory and other symptoms, as well as passive monitoring by the HCWs themselves. If HCWs are exposed to known SARS patients, symptom monitoring should include less specific, early symptoms such as malaise, headache, diarrhea, chills, rigors, rhinorrhea, and sore throat in accordance with the latest SARS case definition. ${ }^{25}$
In addition to performing symptom surveillance of HCWs, facilities should also instruct them on actions to take in the event of an unprotected exposure to a SARS patient. Because of the high risk of transmission, HCWs with an unprotected exposure (eg, during an aerosol-generating procedure) should be removed from patient care for 10 days. However, HCWs with other unprotected exposures need not be removed from duty but should continue to undergo active symptom surveillance.

Patient Surveillance. Hospitalized patients are a third potential reservoir of unrecognized SARS cases in healthcare facilities. Under most circumstances, inpatients will not have contact with known SARS patients, and thus will be at lower risk than HCWs for SARS infection while hospitalized. Therefore, SARS surveillance among patients is recommended only if transmission has occurred in the facility or when a larger number of SARS patients are hospitalized in the facility. When transmission is limited and all cases have clearly defined sources, surveillance efforts should be directed at finding patients who have unexplained fever or lower respiratory tract symptoms. In the setting of hospital-associated transmission with unclear sources, surveillance activities should expand to include any patient with new fever or lower respiratory tract symptoms, including those with apparent explanations for the symptoms. However, even when limited transmission has occurred, HCWs should maintain a high index of suspicion for possible SARS when evaluating patients with nosocomial fever.

\section{Access Control Measures}

Restricting access to the healthcare facility can also help minimize the risk of exposures to unrecognized cases. In the presence of SARS-CoV transmission in the area around the facility, visitors should be limited, perhaps to one per patient per day. This will help to both limit potential exposures in the hospital and facilitate entry screening. If linked transmission occurs in the facility, visitors should be limited regardless of SARS activity in the area around the facility. In addition, the facility should consider limiting elective admissions and procedures to those that are considered high priority. If unlinked transmission is occurring in the facility, all individuals entering the facility are potentially at risk because the source of exposures is unknown. In this setting, the facility should close its emergency department and inpatient wards to new admissions to limit ongoing exposures.

\section{Managing Shortages of AIIRs and Supplies}

Although placement of SARS patients in AIIRs may be possible in most facilities when there are relatively few cases, these resources may be quickly overwhelmed by a larger outbreak. Construction of new AIIRs is one option for increasing the capacity to respond to SARS. However, creation of a "SARS unit" where patients could be cohorted and isolated in the absence of AIIRs is an alternative that can be used especially when the number of cases increases rapidly. 
The $\mathrm{CDC}$ guidance recommends airborne infection isolation whenever possible for SARS patients. Although a dedicated SARS unit will likely use rooms that lack the airhandling advantages conferred by AIIRs, there are potential benefits to this approach. First, a dedicated SARS unit allows SARS-related resources to be concentrated in one area of the hospital, making it possible to designate certain equipment, such as portable x-ray units, for use with SARS patients and thereby reduce the risk of possible fomite transmission. Second, SARS units allow concentration of intensive infection control activities, such as careful monitoring of compliance with precautions and entry screening. Third, designated units allow SARS patients to be physically separated from others in the hospital and facilitate the designation of a relatively limited number of staff to care for only SARS patients, reducing the risks of inadvertent exposures of HCWs. In some facilities, it may be feasible to modify existing wards to create entire units that have negative pressure with respect to surroundings areas, and this option should be explored for any potential SARS unit.

Facilities where AIIRs are widely dispersed or located primarily on floors with immunosuppressed patients may choose to open SARS units even before all available AIIRs are occupied. However, there are some instances in which consideration should still be given to placing patients in AIIRs rather than on a SARS unit. Patients who are known to have infected other individuals prior to admission might be at higher risk to transmit the disease and thus the additional protection conferred by AIIRs may be preferable for protecting HCWs. Also, patients who are under investigation for SARS but who do not meet the SARS case definition should be housed in AIIRs if possible. Many patients under investigation for SARS, perhaps even some who meet the case definition, will not have SARS. Housing these patients under investigation for SARS in AIIRs and not on a SARS unit will help minimize the risks of cross-transmission.

Likewise, facilities will need to consider the current availability of personal protective equipment such as respirators and anticipate how demand for this equipment will change as the number of patients with SARS increases. It is recommended that facilities establish back-up plans in advance in the event that supplies become limited. Supply issues should also be discussed with public health officials to determine what community-level plans exist to address possible shortages.

\section{Managing Staffing and Personnel Challenges}

Facilities should evaluate potential staffing needs for the care of various numbers of SARS patients. If possible, designated teams for the care of SARS patients should be created in advance to provide both routine and emergent care to the initial SARS patients who present to a facility. These teams would learn about SARS infection control procedures in advance, which would allow them to care for patients immediately and also to serve as a resource to train others if the need arises. Consideration will also have to be given to how staffing could be expanded in a more extensive outbreak, such as relocating staff from other areas of the facility. Plans for both initial and ongoing training on infection control measures and the use of personal protective equipment must also be developed. Finally, the guidance document recommends that facilities make plans to provide assistance to $\mathrm{HCWs}$ to help them cope with the stresses of responding to a SARS outbreak including both logistical support and access to mental health professionals.

\section{DISCUSSION}

Individual healthcare facilities should develop plans to manage SARS outbreaks; however, issues will arise that exceed the scope of any one facility and must be addressed at a community, regional, and national level by representatives from healthcare systems, public health, and industry. Although there are no simple solutions to these issues, contributors to the CDC guidance document emphasized that they should be the topics of ongoing discussions as they have important implications not only for SARS but for any emerging infectious emergency. Some of these key issues are raised in the guidance document and include the following:

1. Whether certain facilities should be designated to preferentially house SARS patients.

2. How community-wide SARS evaluation centers might be created.

3 . How new AIIRs might be rapidly built and certified.

4. Ways to provide surge capacity personnel to heavily affected facilities using retired staff or staff from other facilities.

5 . Methods to provide liability coverage and credentials to providers who fill the surge capacity needs.

6. Means to ensure adequate supplies during an outbreak and equitable distribution of the supplies.

7. What emergency funds might be available to assist facilities with construction, personnel, and supply costs as well as lost revenue.

8. The cost-effectiveness of a respiratory hygiene and cough etiquette strategy.

Healthcare facilities are likely to be focal points in future SARS outbreaks should they occur. Experience during the SARS outbreak of 2003 demonstrated that facilities have the potential to serve as the epicenter of SARS transmission in a community but also can play a key role in controlling the outbreak at its earliest stages. Preparedness planning will be essential for helping facilities manage future outbreaks of SARS as well as other emerging and resurgent infectious diseases. Guidance for SARS preparedness and response in healthcare facilities has been developed by the CDC based on experiences from facilities in other countries, tailored for use in the United States. The guidance reflects significant input from members of numerous partner organizations, including public health, healthcare providers, and hospital administration. It is hoped that facilities will be able to use 
this guidance to develop specific, institutional preparedness and response plans that will assist in minimizing the impact of future outbreaks, not just of SARS but of any potential infectious disease emergency.

\section{REFERENCES}

1. World Health Organization. WHO Issues a Global Alert About Cases of Atypical Pneumonia: Cases of Severe Respiratory Illness May Spread to Hospital Staff. Geneva: World Health Organization; 2003. Available at www.who.org.

2. Ksiazek TG, Erdman D, Goldsmith CS, et al. A novel coronavirus associated with severe acute respiratory syndrome. $N$ Engl $J \mathrm{Med}$ $2003 ; 348: 1953-1966$.

3. Ho AS, Sung JJY, Chan-Yeung M. An outbreak of severe acute respiratory syndrome among hospital workers in a community hospital in Hong Kong. Ann Intern Med 2003;139:564-567.

4. Booth CM, Matukas LM, Tomlinson GA, et al. Clinical features and short-term outcomes of 144 patients with SARS in the greater Toronto area. JAMA 2003;289:2801-2809.

5. Anonymous. Severe acute respiratory syndrome: Taiwan, 2003 MMWR 2003;52:461-466.

6. Centers for Disease Control and Prevention. Public Health Guidance for Community-Level Preparedness and Response to Severe Acute Respiratory Syndrome (SARS). Atlanta, GA: Centers for Disease Control and Prevention; 2003. Available at www.cdc.gov/ncidod/sars/sarsprepplan.htm.

7. Varia M, Wilson S, Sarwal S, et al. Investigation of a nosocomial outbreak of severe acute respiratory syndrome (SARS) in Toronto, Canada. Canadian Medical Association Journal 2003;169:285-292.

8. Anonymous. Update: severe acute respiratory syndrome-Toronto, Canada, 2003. MMWR 2003;52:547-550.

9. Lingappa JR, McDonald LC, Simone P, Parashar UD. Wrestling SARS from uncertainty. Emerg Infect Dis 2004;10:167-170.

10. Farquharson C, Baguley K. Responding to the severe acute respiratory syndrome (SARS) outbreak: lessons learned in a Toronto emergency department. Journal of Emergency Nursing 2003;29:222-228.

11. Gopalakrishna G, Choo P, Leo YS, et al. SARS transmission and hospital containment. Emerg Infect Dis 2004;10:395-400.

12. World Health Organization. Consensus Document on the Epidemiology of Severe Acute Respiratory Syndrome. Geneva: World Health Organization; 2003. Available at www.who.int/csr/sars/en/
WHOconsensus.pdf.

13. Seto WH, Tsang D, Yung RWH et al. Effectiveness of precautions against droplets and contact in the prevention of nosocomial transmission of severe acute respiratory syndrome (SARS). Lancet 2003:361:1519-1520.

14. Anonymous. Cluster of severe acute respiratory syndrome cases among protected health care workers: Toronto, Canada, April 2003. MMWR 2003;52:433-436.

15. Anonymous. Severe acute respiratory syndrome: Singapore, 2003. MMWR 2003;52:405-411.

16. Ignatius TS, Yu MB, Yuguo L, et al. Evidence of airborne transmission of the severe acute respiratory syndrome virus. N Engl J Med 2004;350:17311739 .

17. Fraser VJ, Johnson K, Primack J, Jones M, Medoff G, Dunagan WC. Evaluation of rooms with negative pressure ventilation used for respiratory isolation in seven midwestern hospitals. Infect Control Hosp Epidemiol 1993;14:619-622.

18. Srinivasan A, Jernigan D, Liedyke L, Strausbaugh L. SARS preparedness plans and infrastructure in the United States: a survey of infectious disease specialists. Presented at the 41st Annual Meeting of the Infectious Diseases Society of America; October 9-12, 2003; San Diego, CA. Abstract LB-7.

19. McDonald LC, Simore A, Su IJ, et al. SARS in healthcare facilities: Toronto and Taiwan. Emerg Infect Dis 2004;10:777-781.

20. Lau JTF, Fung KS, Wong TW, et al. SARS transmission among hospital workers in Hong Kong. Emerg Infect Dis 2004;10:280-286.

21. Anonymous. Will your ED have staff quarantined for SARS? Brace yourself for the worst. ED Management 2003;15(suppl 1):49-51.

22. Centers for Disease Control and Prevention. Guideline for Isolation Precautions in Hospitals. Atlanta, GA: Centers for Disease Control and Prevention; 1994. Available at www.cdc.gov/ncidod/hip/ ISOLAT/Isolat.htm.

23. Centers for Disease Control and Prevention. Guidelines for preventing the transmission of Mycobacterium tuberculosis in healthcare facilities: 1994. Atlanta, GA: Centers for Disease Control and Prevention; 1994 Available at www.cdc.gov/mmwr/preview/mmwrhtml/00035909.htm.

24. Karanfil LV, Conlon M, Lykens K, et al. Reducing the rate of nosocomially transmitted respiratory syncytial virus. Am J Infect Control 1999;27:91-96.

25. Anonymous. Revised U.S. surveillance case definition for severe acute respiratory syndrome (SARS) and update on SARS cases United States and worldwide, December 2003. MMWR 2003;52:1202-1206.

\title{
Medical News
}

\author{
Edited by Gina Pugliese, RN, MS; Martin S. Favero, PhD
}

\section{Are Hospital Surfaces Reservoirs for Nosocomial Infection?}

Hota from the University Medical Center, Chicago, reviewed the subject of environmental transmission of infections that originate from the environment. He pointed out that despite documentation that the inanimate hospital environment (eg, surfaces and medical equipment) becomes contaminated with nosocomial pathogens, the data that suggest that contaminated fomites lead to nosocomial infections do so indirectly. Pathogens for which there is more compelling evidence of survival in environmental reservoirs include Clostridium difficile, vancomycin-resistant enterococci, and methicillin-resistant Staphylococcus aureus, and pathogens for which there is evidence of probable survival in environmental reservoirs include norovirus, influenza virus, severe acute respiratory syndrome-associated coronavirus, and Candida species. Strategies to reduce the rates of nosocomial infection with these pathogens should conform to established guidelines, with an emphasis on thorough environmental cleaning and use of Environmental Protection Agency-approved detergent-disinfectants.

FROM: Hota B. Contamination, disinfection, and cross-colonization: are hospital surfaces reservoirs for nosocomial infection? Clin Infect Dis 2004;39:1182-1189. 